\title{
DETERMINING THE BEST METHOD FOR NELLCOR PULSE OXIMETER SENSOR APPLICATION
}

\author{
J.A. Dawson ${ }^{1,2}$, A. Saraswat ${ }^{3}$, L. Simionato ${ }^{3}$, M. Thio ${ }^{1}$, C.O.F. Kamlin ${ }^{1,3}$, L. Owen ${ }^{1}$, G.M. Schmoelzer ${ }^{1}$, P.G. \\ Davis $^{1,2,3}$ \\ ${ }^{I}$ Neonatal Services, The Royal Women's Hospital, ${ }^{2}$ The Murdoch Childrens Research Institute, ${ }^{3}$ The \\ University of Melbourne, Melbourne, VIC, Australia
}

Background and aims: To be useful in the delivery room, pulse oximeters must provide clinicians with accurate data in the first minutes after birth. This study aimed to identify the sensor application technique which gave the fastest display of accurate data using the Nellcor N-600x Oximax Pulse Oximeter (PO).

Method: Stable infants from our Neonatal Intensive and Special Care nurseries with electrocardiograph (ECG) monitoring were included. Three sensor application techniques were studied:

(1) sensor connected to cable, then applied to infant;

(2) sensor connected to cable, applied to investigator's finger, then to infant;

(3) sensor applied to infant, then connected to cable.

The order of techniques was randomised. Time taken to apply PO sensor, to display any data, and to display accurate data $\left(\mathrm{HR}_{\mathrm{PO}}=\mathrm{HR}_{\mathrm{ECG}} \pm 3 \mathrm{bpm}\right)$ were recorded using a stopwatch. Means were compared using paired t tests.

Results: Forty infants were studied [mean(SD) birth weight 1455(872) g, gestational age 31(4) weeks, postnatal age 34(4) weeks]. Time to acquire any data mean(SD): method 1: 15(13) s; method 2: 13(6) s; method 3: 9(7) s. Method 3 acquired data significantly faster than methods $1(\mathrm{p}=0.013)$ and $2(\mathrm{p}=0.004)$. Time to acquire accurate data mean(SD): method 1 17(13) s; method 2 14(7) s; method 3 11(9) s. Method 3 acquired accurate data significantly faster than method $1(\mathrm{p}=0.016)$, but not method $2(\mathrm{p}=0.15)$.

Conclusions: Applying the N-600x PO sensor to the infant before connecting it to the cable yields fastest acquisition of accurate data. 